\title{
On the need of opening up crowdsourced emergency management systems
}

\author{
Marco Avvenuti · Stefano Cresci - Fabio Del \\ Vigna - Maurizio Tesconi
}

Received: February, 2016

\begin{abstract}
Nowadays, social media analysis systems are feeding on user contributed data, either for beneficial purposes, such as emergency management, or for user profiling and mass surveillance. Here, we carry out a discussion about the power and pitfalls of public accessibility to social media-based systems, with specific regards to the emergency management application EARS (Earthquake Alert and Report System). We investigate whether opening such systems to the population at large would further strengthen the link between communities of volunteer citizens, intelligent systems, and decision makers, thus going in the direction of developing more sustainable and resilient societies. Our analysis highlights fundamental challenges and provides interesting insights into a number of research directions with the aim of developing human-centered social media-based systems.
\end{abstract}

Keywords Social sensing, social media mining, collective intelligence, emergency management, crisis informatics

\footnotetext{
Marco Avvenuti

University of Pisa, Department of Information Engineering, Pisa, Italy

E-mail: marco.avvenuti@unipi.it

Stefano Cresci $(\bowtie)$

National Research Council (CNR), Institute of Informatics and Telematics (IIT)

via G. Moruzzi 1, 56124, Pisa, Italy

Tel.: +39050315 8272, Fax: +39050315 2593;

E-mail: stefano.cresci@iit.cnr.it

Fabio Del Vigna $(\varangle)$

National Research Council (CNR), Institute of Informatics and Telematics (IIT)

via G. Moruzzi 1, 56124, Pisa, Italy

Tel.: +39050315 3322, Fax: +39050 315 2593; and

University of Pisa, Department of Information Engineering, Pisa, Italy

E-mail: fabio.delvigna@iit.cnr.it
}

Maurizio Tesconi

National Research Council (CNR), Institute of Informatics and Telematics (IIT), Pisa, Italy

E-mail: maurizio.tesconi@iit.cnr.it 


\section{Introduction}

Both natural and man-made disasters such as earthquakes, tornadoes, floods, and terrorists attacks, are events that deeply impact wide areas, affecting population and goods, and shaking societies on both economical as well as psychological aspects. These kinds of events usually unchain people involvement in participation and reporting (Vieweg et al., 2008). In fact, in recent years, a significant increment of social media (SM) activity has been observed in the aftermath of mass convergence and emergency events (Bagrow et al., 2011). To this regard, microblogs such as Twitter, Weibo, and Instagram are privileged channels of information diffusion because of their ubiquity and simplicity (Hughes \& Palen, 2009). During emergencies people usually report their experience on these media, which are consequently overwhelmed by information concerning the unfolding scenario. Also, messages shared on these media are often complemented by comments, images, or videos (Roche et al., 2013). Such astonishing amount of data, when collected and aggregated, converge around meaningful information that can be used for decision making (Avvenuti et al., 2015).

As a matter of fact, so far civil protection agencies do not fully exploit tools based on SM contents and still heavily rely only on traditional technologies (e.g., seismographic networks, on-the-ground surveys, aerial or satellite images) in the most intense phases of emergency response (Avvenuti, Cresci, Del Vigna, \& Tesconi, 2016). What happened during recent major disasters, such as the Tohoku earthquake and tsunami (Japan 2011), the Emilia earthquake (Italy 2012) and the Himalayan earthquake (Nepal 2015), highlighted a divide between the promising results and technologies proposed by Academia and the traditional technologies still employed by the majority of emergency responders. Therefore, fundamental questions arise: how can we strengthen the link between intelligent systems (Russell et al., 2003), new communication media and societies? Furthermore, can systems based on SM data analysis be accessible to everyone? Do they really improve the quality of the assessment and the control during emergencies?

Contribution. Here, we tackle these challenges by discussing the opportunities and the drawbacks of opening novel emergency management systems to the public. We consider a real case study, the EARS (Earthquake Alert and Report System) system (Avvenuti, Cresci, Marchetti, et al., 2014), developed by IIT-CNR ${ }^{1}$ for civil protection agencies to support population in crisis scenarios using data gathered from social media, Twitter primarily. Given the importance of crowd-contributed data in the process of emergency response, we wonder what would happen in case tools and applications for social media mining were made available to the population at large. Building on the EARS experience, we discuss positive and negative aspects of such possibility, as well as benefits from a collective point of view. Our discussion takes into account benefits for those who become more engaged in the analysis process (Del Vigna, Petrocchi, et al., 2016), possible drawbacks such as false alarms, intentional attacks, and profiling of users.

1 http://www.iit.cnr.it/en 


\section{Collective intelligence and wisdom of the crowds in emergency} management

During the last years, several applications feeding on SM data have been developed with the purpose of event detection, disaster response (Avvenuti, Cresci, Marchetti, et al., 2016), business intelligence, health monitoring (Del Vigna, Avvenuti, et al., 2016), cybercrime fight (Cresci et al., 2016), and situational awareness (Cresci, Cimino, et al., 2015). Among these, are those applications designed to provide support in the aftermath of mass-emergency situations. In fact, the spontaneous production of content-rich and actionable information can be a valuable support towards the rapid understanding of the emergency scenario, as recently demonstrated by a number of works that specifically focused on crisis management issues (Sakaki et al., 2013; Earle et al., 2012; Yin et al., 2012; Avvenuti, Cresci, La Polla, et al., 2014; Middleton et al., 2014; Cresci, Tesconi, et al., 2015). Moreover, the convergence of collective opinions and "voice", namely "wisdom of the crowds", reveals to be significant when there is no other available data source, as it typically happens in crisis scenarios. This convergence towards statistical meaningful information was exploited in all these works by searching for spontaneous emergency reports shared on social media, in order to detect outbreaking emergencies and improve situational awareness through "intelligent systems".

An intelligent system is a machine, powered by artificial intelligence and machine learning techniques, that has the capacity to gather and analyze data, and to communicate with other systems (Russell et al., 2003). Within these systems, humans play an important, yet rather marginal, role. Indeed, volunteer citizens are solely considered as data sources or "social sensors", namely "humans as citizens on the ubiquitous Web, acting as sensors and sharing their observations and views using mobile devices and Web 2.0 services" (Sheth, 2009).

Intelligent systems employed in the emergency management field have been originally designed to support decision makers and, usually, are not publicly accessible. Therefore, those social sensors that contributed data to the systems with their volunteered observations, may feel the frustration of not directly benefiting from their efforts, nor seeing the results of the analysis performed by such systems. Indeed, social sensors are not even part of the analysis process, as these intelligent systems are fully data-driven and automated, and their output is the result of complex algorithms and data analysis techniques. Recent research proposed instead to increment the involvement of people, in order to try and merge human- and machine-computation, thus going in the direction of reducing the gap between volunteer citizens and intelligent systems and technologies (Imran et al., 2014; Vieweg \& Hodges, 2014).

This brief analysis outlines that there is still a rather big divide between volunteer citizens, analysis systems, and emergency stakeholders. It highlights how a deeper and more effective synergy between these worlds might be the key for developing more resilient and human-centered systems (Gill, 2013; A. Kavanaugh et al., 2013). Conversely, following the current paradigm of seeing humans only as ubiquitous data sources, might lead to the opposite direction of mass surveilled societies (Gill, 2013). This issue is particularly relevant within the context of terrorism and crime fight (Ball \& Webster, 2003). Indeed, the recent rise of terrorist attacks and global violence pushed an increasing number of people, from decision 


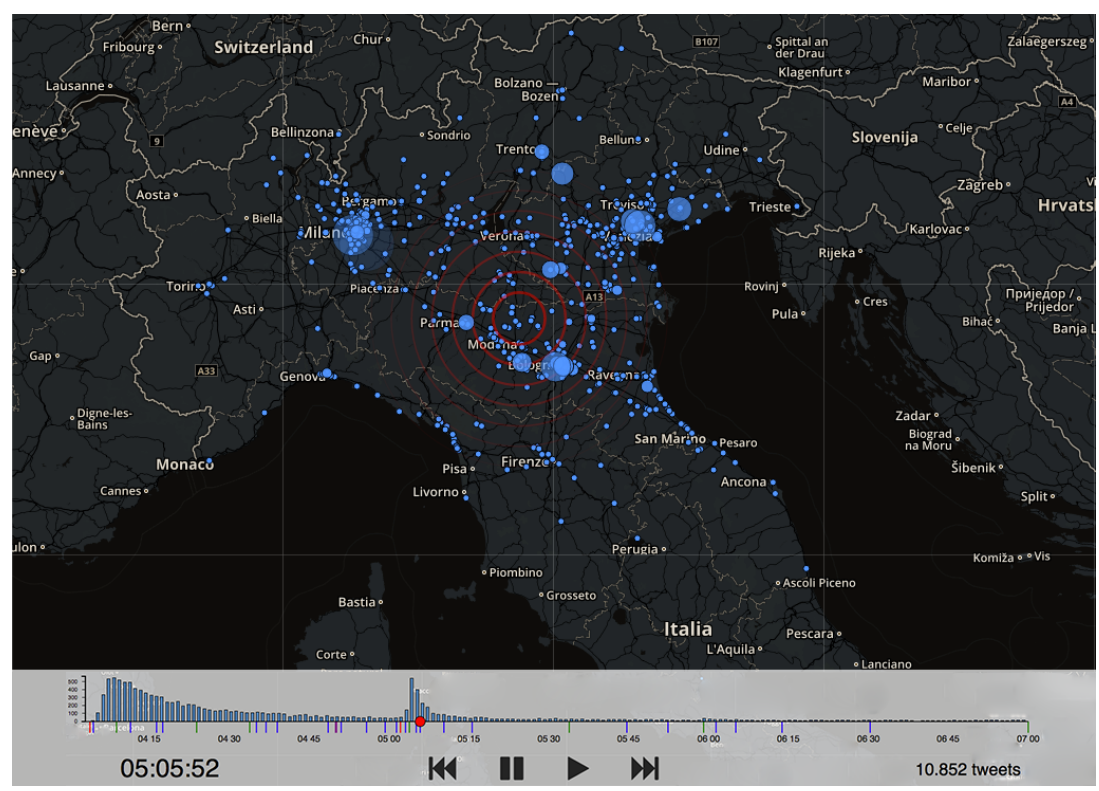

Fig. 1 Excerpt of the EARS web interface. The system shows a geographic map of an earthquake-stricken region where geolocated tweets are represented as blue dots. The map is interactive and every dot (i.e., every tweet) can be clicked and expanded in order to see its content. The bottom part of the interface features a timeline of relevant events. Such events are displayed as colored milestones below the time axis. A bar chart representing the number of tweets per minute is shown above the time axis. An interactive time slider allows users to pick a specific moment in time and display on the map only the tweets shared until that time.

makers to normal citizens, into considering mass surveillance as a possible solution to these problems ${ }^{2}$.

\section{The EARS system}

In the aftermath of disasters, massive spontaneous messages are shared among SM communities. The convergence about specific topics creates statistical meaningful divergence from typical frequencies, and abnormal quantities of messages typically are caused by events. Thus, quality of the results is strongly affected by people participation and collaboration in sharing information and exchanging messages during crisis events. Dramatic events shake public opinion rapidly and trigger people participation to discussions. Detecting peaks of activity in SM, like EARS does, can lead to the detection of crises. Moreover, the emotional impact of crises is typically high and people feel particularly involved. In fact, people that are close to stricken areas, like witnesses, are prone to share details about their surroundings.

EARS is an opportunistic crowdsourced Twitter-based system that aims at helping responders in understanding unfolding emergencies (Avvenuti, Cresci, Marchetti, et al., 2014). The system performs several tasks such as (i) data col-

2 https://www. theguardian.com/us-news/2015/apr/22/mass-surveillance-needed-isis-attack -mike-rogers 
lection, (ii) data filtering, (iii) event detection, (iv) damage assessment, and (v) data visualization. The data collection task is performed by means of several opportunistic Twitter crawlers. The crawlers exploit Twitter APIs and a set of emergency-related keywords in order to collect data in real-time. By following an opportunistic approach, EARS captures a wide variety of information - rich in heterogeneity and spontaneity - at the expense of increased noise in the content and of requiring the system to handle high volumes of unstructured text. Because of the noise in collected data, the systems also performs a data filtering task in order to retain only the most relevant tweets. This task is carried out by exploiting a natural language processing machine learning classifier (Avvenuti, Cresci, Marchetti, et al., 2014). Relevant data is then fed to a burst detection algorithm in order to perform the event detection task. The algorithm is capable of spotting exceptional levels of conversation, represented by bursts of messages, about different emergencies. EARS is capable of detecting emergency situations with high precision and recall (e.g., it recognized more than $80 \%$ of earthquakes with magnitude $\geq 3.5$ degrees) (Avvenuti, Cresci, Marchetti, et al., 2014). The system sends reports as well as emails and Twitter notifications for every detected event. Statistical algorithms, such as regression models, are employed to produce an assessment of the extent of damage in the aftermath of severe emergencies. Finally, the system also generates interactive crisis maps showing the areas the are likely to have suffered significant damage (Avvenuti, Cresci, Del Vigna, \& Tesconi, 2016). Both the crisis maps and the other results of the analyses are accessible via a Web interface, as shown in Figure 1. The impromptu damage assessment, the crisis maps, and the reports generated by EARS allow decision makers to better understand the unfolding scenario and evaluate the best strategy for prioritization of resources and their allocation (Avvenuti, Cresci, Del Vigna, \& Tesconi, 2016).

Since the EARS system became stable and fully deployed, we started wondering whether the crowd really benefited from applications like EARS. The experience gained in this project, as well as the analysis of other similar systems such as (Sakaki et al., 2013; Earle et al., 2012; Yin et al., 2012; Middleton et al., 2014), led us to the conclusion that opportunistic systems can exploit huge quantities of information to detect unfolding emergencies, but actually exclude the crowds from the processes of data analysis and interpretation. This can lead to a looser coupling between crowds and crowd-enabled technology. Furthermore, systems that monitor social media contents may be perceived as tools for mass control, instead of a support. In the following we carry out a discussion on the effects of publicly opening crowdsourced emergency management systems to the population at large.

\section{The pros and cons of an open emergency management intelligent system}

The majority of crowdsourced emergency management systems, such as (Sakaki et al., 2013; Earle et al., 2012; Yin et al., 2012; Avvenuti, Cresci, Marchetti, et al., 2014; Middleton et al., 2014; Avvenuti, Cresci, Del Vigna, \& Tesconi, 2016), were designed to be of support for decision makers, keeping in mind that final users would have been members of a civil protection agency or experts in responding to emergencies. While developing and fine-tuning the EARS system, we debated whether a more transparent handling of the extracted information could also be of direct benefit for the population at large, well aware that several concerns may 
arise. Such concerns are mainly related to the possible misinterpretation of the information made available by the systems and to possible malicious behaviors.

Monitoring authorities. Social media are becoming monitoring tools that encourage collective intelligence. In fact, an efficient bidirectional communication between population and institutions is fundamental to improve emergency response. This cooperation can be enhanced by exploiting social media in order to allow citizens to forward public requests for help and to notify dangers and critical situations. Publicly opening SM-based emergency management systems, on the one hand, would allow the population at large to better understand the kind and the volume of information made available to decision makers. On the other hand, this would stimulate civil protection agencies to better exploit information extracted from social media (e.g., ensuring that crowd generated reports were timely taken into account). As a consequence, institutions would be forced to improve the service quality, being aware that the effectiveness of their actions is verifiable, while crowds would be better motivated to share information that can be potentially straight available and verifiable by everyone.

Citizen empowerment. An open SM-based emergency management system makes digital volunteers aware that the information they share can directly help emergency responders and guide their decisions. This self consciousness fosters the participation of the crowd in discussions and promotes proactivity in documenting the unfolding scenario posting tweets, photos, and videos. This form of citizen empowerment is likely to lead to a virtuous circle in which digital volunteers ${ }^{3}$ are encouraged to share more and better information for decision makers to act. The active participation of people in emergency response is beneficial also in case of terrorist attacks, to help identifying attackers, or in massive events, to monitor the situation and maintain sufficient services availability. Only recently, a few works envisioned this possibility and started experimenting with humans carrying out part of the analyses, or being actively involved in the dissemination of the results (Imran et al., 2014; Vieweg \& Hodges, 2014). It is still too early to tell whether these approaches will ultimately succeed or fail, but the growing trend of increasing citizens involvement is surely fostering the development of increasingly human-centered societies.

False alarms. It is the case, however, that opening this kind of systems to the public at large also poses some serious questions about their trustworthiness and credibility (Castillo et al., 2011). SM systems are usually evaluated on their recall performance (i.e., the ability to recognize relevant events), while their precision (i.e., the ability to minimise false detections) is often overlooked (Avvenuti, Cimino, et al., 2016). Frequent false detections will inevitably lead people to ignore the system's outcome, making it unreliable and, at some level, useless. In some circumstances, false detections may even create alarms that induce panic and fear in populations. Errors are often caused by a poor quality of data source, as SM outputs are subject to people operating in extreme conditions: messages shared during emergencies are fragmented, and lack a defined structure and means to assess information trustworthiness and credibility (A. L. Kavanaugh et al., 2012).

3 Examples of organizations that currently employ digital volunteers and that define their role in modern emergency management are the Humanitarian Response (https:// ww. humanitarianresponse.info/en/applications/tools/category/digital-volunteers) and the International Red Cross (http://redcrosschat.org/disaster-digital-volunteer -training/\#sthash.yVdEzS21.dpbs) 
We argue that system quality may be improved by putting the crowd-in-the-loop, i.e., allowing people to autonomously apply corrections to the system. In fact, statistical results show that the aforementioned "wisdom of the crowds" ensures the absence of errors in data, or contributes to its reduction. Collaborative projects like Wikipedia ${ }^{4}$, HarassMap ${ }^{5}$, Humanitarian Tracker ${ }^{6}$, and Ushahidi ${ }^{7}$ are just but a few examples of "open" platforms that benefit from an active citizen participation. As such, those systems are particularly suitable to carry out verification and corroboration of user contributed reports and might serve as compelling examples for future developments of SM-based emergency management systems.

Attack resilience. False detections might also be caused by malicious users willing to purposely disrupt the service. Robustness to attacks in critical systems, such as those operating in emergency management, is mandatory and should become a primary guideline in system design. Data filtering, a cleaning process carried out in many existing systems (Sakaki et al., 2013; Earle et al., 2012; Yin et al., 2012; Middleton et al., 2014), helps in validating single messages, but it fails to safeguard the system from bursts of bogus messages purposely shared. Although this issue is well-known to social media researchers, to date the vast majority of SM-based emergency management systems does not employ security mechanisms (Avvenuti, Cimino, et al., 2016). In the EARS project, we experimented with fake accounts detection algorithms in order to reduce security concerns and mitigate the problem of bursts of fictitious (i.e., fake) messages (Cresci, Di Pietro, et al., 2015). Fake accounts detection and removal represents just one among the possible strategies for protecting SM-based systems from malicious attacks. Indeed, more research and experimentation is needed in this direction so as to increase the resilience and the reliability of these critical systems.

Centralized vs. distributed emergency management. One of the key points of discussion concerns who should really manage and organize emergency response and communications. A centralized approach has the drawback of a lack of finegrained presence on struck areas. Indeed, it is unfeasible for civil protection agencies to continuously and accurately monitor the territory. Moreover, emergency responders are slow in the adoption of systems that differ from those traditionally used due to authoritative and responsibility issues. This position potentially hinders the amount of information available to decision makers and makes them not completely aware of the situation when reacting to a crisis. Whilst relying on the crowds can allow task parallelization, the lack of a central authority may conversely deteriorate decision quality. Furthermore, citizens often lack technical skills and thus efforts might be inhibited by a lack of competences. Nonetheless, in recent emergencies, volunteer citizens converging to the disaster zone played a fundamental role in starting and maintaining grassroot initiatives, as it happened in Genoa after a recent flash flood ${ }^{8}$, where volunteers, called "mud angels", helped to remove mud from the streets, without any external coordination ${ }^{9}$. In this light,

\footnotetext{
4 https://en.wikipedia.org

5 http://harassmap.org/en/

6 http://www.humanitariantracker.org/

7 https://www. ushahidi.com/

8 https://it.wikipedia.org/wiki/Alluvione_di_Genova_del_9_e_10_ottobre_2014 (in Italian)

9 https://en.wikipedia.org/wiki/1966_flood_of_the_Arno_River
} 
opening emergency management systems to these volunteers would give them tools to improve their coordination and efforts.

\section{Conclusions and future directions}

The possibility to rely on publicly available data shared on social media platforms, allows intelligent systems to extract knowledge that is increasingly important for monitoring and intelligence purposes. As highlighted in our discussion, which was focused on the emergency management field, the growing importance of such usergenerated data poses serious questions about the role and the centrality of humans (and the data they produce) in modern intelligent systems. A first dichotomy arises by considering the context in which such data is produced. Indeed, access to private information for the common good could be desirable in some situations, but it also may also represent a threat to citizen privacy if used for mass surveillance. For instance, during natural disasters users might be willing to disclose more information about themselves and to loosen their privacy requirements, since sensitive information is likely to be used for beneficial purposes (e.g., to track last known position of missing people). Anyway, this might not be the case during electoral campaigns when citizen personal information and opinions can be exploited to infer vote intentions or to assess the dissent towards a political party. Even more worrying is the possibility to mass surveil online user activities and private interactions (e.g., chat or phone conversations) in the name of purported intelligence purposes, such as crime or terrorism prevention. Therefore, user perception of the common good and of the purpose for which their data is being collected and analyzed, might be crucial to motivate them to disclose such data, thus enabling "social" intelligent systems to perform their tasks.

A possible solution for putting back citizens at the core of modern intelligent systems, instead of being relegated only to the borders of such systems as mere data sources, is to allows them to directly benefit from the results of those systems. Anyway although appealing, the idea of feeding results back to the citizens has never been implemented in already deployed systems. In order to fill this gap, designers of the next-generation of "social" intelligent systems should be imaginative to this regard, and could take inspiration from the research and applications already developed in the fields of e-democracy and e-government (Coleman \& Blumler, 2009). However, publicly opening the results of modern intelligent systems to the population at large also requires to solve a number of challenges, such as ensuring the trustworthiness and credibility of both the collected and disclosed information, managing the decentralized coordination of information-empowered citizens, and more. All these issues, analyzed and discussed in this manuscript, pose interesting research challenges and represent promising directions of experimentation for the future.

\section{References}

Avvenuti, M., Cimino, M. G., Cresci, S., Marchetti, A., \& Tesconi, M. (2016). A framework for detecting unfolding emergencies using humans as sensors. SpringerPlus, 5(1), 1. 
Avvenuti, M., Cresci, S., Del Vigna, F., \& Tesconi, M. (2016). Impromptu crisis mapping to prioritize emergency response. Computer, 49(5), 28-37.

Avvenuti, M., Cresci, S., La Polla, M., Marchetti, A., \& Tesconi, M. (2014). Earthquake emergency management by social sensing. In Pervasive Computing and Communications Workshops (PERCOM Workshops), 2014 IEEE International Conference on.

Avvenuti, M., Cresci, S., Marchetti, A., Meletti, C., \& Tesconi, M. (2014). EARS (Earthquake Alert and Report System): A real time decision support system for earthquake crisis management. In Proceedings of the 20th ACM SIGKDD International Conference on Knowledge Discovery and Data Mining. ACM.

Avvenuti, M., Cresci, S., Marchetti, A., Meletti, C., \& Tesconi, M. (2016). Predictability or early warning: Using social media in modern emergency response. IEEE Internet Computing, 20(6), 4-6.

Avvenuti, M., Del Vigna, F., Cresci, S., Marchetti, A., \& Tesconi, M. (2015). Pulling information from social media in the aftermath of unpredictable disasters. In Information and Communication Technologies for Disaster Management (ICT-DM), 2015 IEEE 2nd International Conference on (pp. 258-264).

Bagrow, J. P., Wang, D., \& Barabasi, A.-L. (2011). Collective response of human populations to large-scale emergencies. PloS one.

Ball, K., \& Webster, F. (2003). The intensification of surveillance: Crime, terrorism and warfare in the information age. Pluto Press.

Castillo, C., Mendoza, M., \& Poblete, B. (2011). Information credibility on twitter. In Proceedings of the 20th international conference on World Wide Web (pp. 675-684).

Coleman, S., \& Blumler, J. G. (2009). The internet and democratic citizenship: Theory, practice and policy (Vol. 1). Cambridge University Press.

Cresci, S., Cimino, A., Dell'Orletta, F., \& Tesconi, M. (2015). Crisis mapping during natural disasters via text analysis of social media messages. In International Conference on Web Information Systems Engineering-WISE 2015 (pp. 250-258).

Cresci, S., Di Pietro, R., Petrocchi, M., Spognardi, A., \& Tesconi, M. (2015). Fame for sale: efficient detection of fake Twitter followers. Decision Support Systems, 80, 56-71.

Cresci, S., Di Pietro, R., Petrocchi, M., Spognardi, A., \& Tesconi, M. (2016). Dnainspired online behavioral modeling and its application to spambot detection. IEEE Intelligent Systems, 31(5), 58-64.

Cresci, S., Tesconi, M., Cimino, A., \& Dell'Orletta, F. (2015). A linguisticallydriven approach to cross-event damage assessment of natural disasters from social media messages. In Proceedings of the 24th international conference companion on World Wide Web (pp. 1195-1200).

Del Vigna, F., Avvenuti, M., Bacciu, C., Deluca, P., Petrocchi, M., Marchetti, A., \& Tesconi, M. (2016). Spotting the diffusion of new psychoactive substances over the internet. In International Symposium on Intelligent Data Analysis (pp. 86-97).

Del Vigna, F., Petrocchi, M., Tommasi, A., Zavattari, C., \& Tesconi, M. (2016). Semi-supervised knowledge extraction for detection of drugs and their effects. In International Conference on Social Informatics (pp. 494-509).

Earle, P. S., Bowden, D. C., \& Guy, M. (2012). Twitter earthquake detection: earthquake monitoring in a social world. Annals of Geophysics, 54(6), 708- 
715.

Gill, K. S. (2013). Citizens and netizens: a contemplation on ubiquitous technology. AI \& SOCIETY, 28(2), 131-132.

Hughes, A. L., \& Palen, L. (2009). Twitter adoption and use in mass convergence and emergency events. International Journal of Emergency Management, 6(34), 248-260.

Imran, M., Castillo, C., Lucas, J., Meier, P., \& Vieweg, S. (2014). Aidr: Artificial intelligence for disaster response. In Proceedings of the companion publication of the 23rd international conference on World Wide Web companion (pp. 159$162)$.

Kavanaugh, A., Ahuja, A., Pérez-Quiñones, M., Tedesco, J., \& Madondo, K. (2013). Encouraging civic participation through local news aggregation. In Proceedings of the 14th Annual International Conference on Digital Government Research (pp. 172-179).

Kavanaugh, A. L., Fox, E. A., Sheetz, S. D., Yang, S., Li, L. T., Shoemaker, D. J., ... Xie, L. (2012). Social media use by government: From the routine to the critical. Government Information Quarterly, 29(4), 480 - 491.

Middleton, S. E., Middleton, L., \& Modafferi, S. (2014). Real-time crisis mapping of natural disasters using social media. IEEE Intelligent Systems, 29(2), 9-17.

Roche, S., Propeck-Zimmermann, E., \& Mericskay, B. (2013). Geoweb and crisis management: Issues and perspectives of volunteered geographic information. GeoJournal, 78(1), 21-40.

Russell, S. J., Norvig, P., Canny, J. F., Malik, J. M., \& Edwards, D. D. (2003). Artificial intelligence: a modern approach (Vol. 2). Prentice hall Upper Saddle River.

Sakaki, T., Okazaki, M., \& Matsuo, Y. (2013). Tweet analysis for real-time event detection and earthquake reporting system development. IEEE Transactions on Knowledge and Data Engineering (TKDE), 25(4), 919-931.

Sheth, A. P. (2009). Citizen sensing, social signals, and enriching human experience. IEEE Internet Computing, 13(4), 87.

Vieweg, S., \& Hodges, A. (2014). Rethinking context: Leveraging human and machine computation in disaster response. Computer, 47(4), 22-27.

Vieweg, S., Palen, L., Liu, S. B., Hughes, A. L., \& Sutton, J. (2008). Collective intelligence in disaster: An examination of the phenomenon in the aftermath of the 2007 virginia tech shootings. In Proceedings of the Information Systems for Crisis Response and Management Conference (ISCRAM).

Yin, J., Lampert, A., Cameron, M., Robinson, B., \& Power, R. (2012). Using social media to enhance emergency situation awareness. IEEE Intelligent Systems, 27(6), 52-59. 